\section{Der Wachtelberg am Mühlbachtal}

\author{
Jens Müller
}

Zwischen Schmölen und Dehnitz, südlich von Wurzen, durchbricht die Mulde auf ca. $112 \mathrm{~m}$ NN den letzten Granitporphyrriegel am Wachtelberg $(147 \mathrm{~m})$, ehe sie hinter Wurzen in die fast tischebene, bis vier Kilometer breite Auenlandschaft mit zahlreichen Flussschlingen, Altwässern und Lachen übergeht. Der Wachtelbergturm erhebt sich markant über die umgebende Landschaft. Hier befinden wir uns in einem der ältesten Naturschutzgebiete (NSG) Sachsens. Das NSG hat eine Größe von 23 ha und liegt auf einer Höhe von 121 bis 147 m NN, dessen Kernbereich die Felskuppe des Wachtelberges mit seiner Trockenrasenflur ist. Umgeben ist sie von Ackerflächen und im Süden schließen sich die Feuchtwiesen im naturnahen Mühlbachtal an. Das Gebiet ist Bestandteil des Landschaftsschutzgebiets Mittlere Mulde. Am Wachtelberg selbst wurde lange Granitporphyr abgebaut, der letzte Steinbruch schloss in den 1930er Jahren. Rund um die Kuppe wurde immer schon Vieh geweidet.
Markant bekrönt die Höhe der Wachtelbergturm, der eigentlich ein Bismarckturm ist. Nach der Entlassung des Reichskanzlers Otto von Bismarck 1890 und nach dessen Tod 1898 setzte in Deutschland eine beispiellose Bismarck-Verehrung ein. Vor allem Denkmäler, meist in Form von Bismarcktürmen, wurden zu seinem Ehren errichtet. In Wurzen ging die Initiative vom Reichsverein für Wurzen und Umgebung unter Vorsitz des Maschinenfabrikanten Richard Klinkhardt aus, der einen solchen Bau anregte. Unter Klinkhardts Vorsitz wurde schließlich ein Komitee für die Errichtung des Turmes gegründet. Es wurde das Grundstück auf dem Wachtelberg erworben und Geld gesammelt, darunter vor allem Spenden, und auch eine Bismarckturm-Lotterie wurde veranstaltet. Als Architekten beauftragte man nach einer Ausschreibung Wilhelm Kreis, dessen von der Deutschen Studentenschaft im Jahr 1899 preisgekrönter Entwurf „Götterdämmerung" bis 1911 insgesamt 47-mal gebaut wur-
Wachtelberg mit Bismarckturm Foto: Wolfgang Ebert

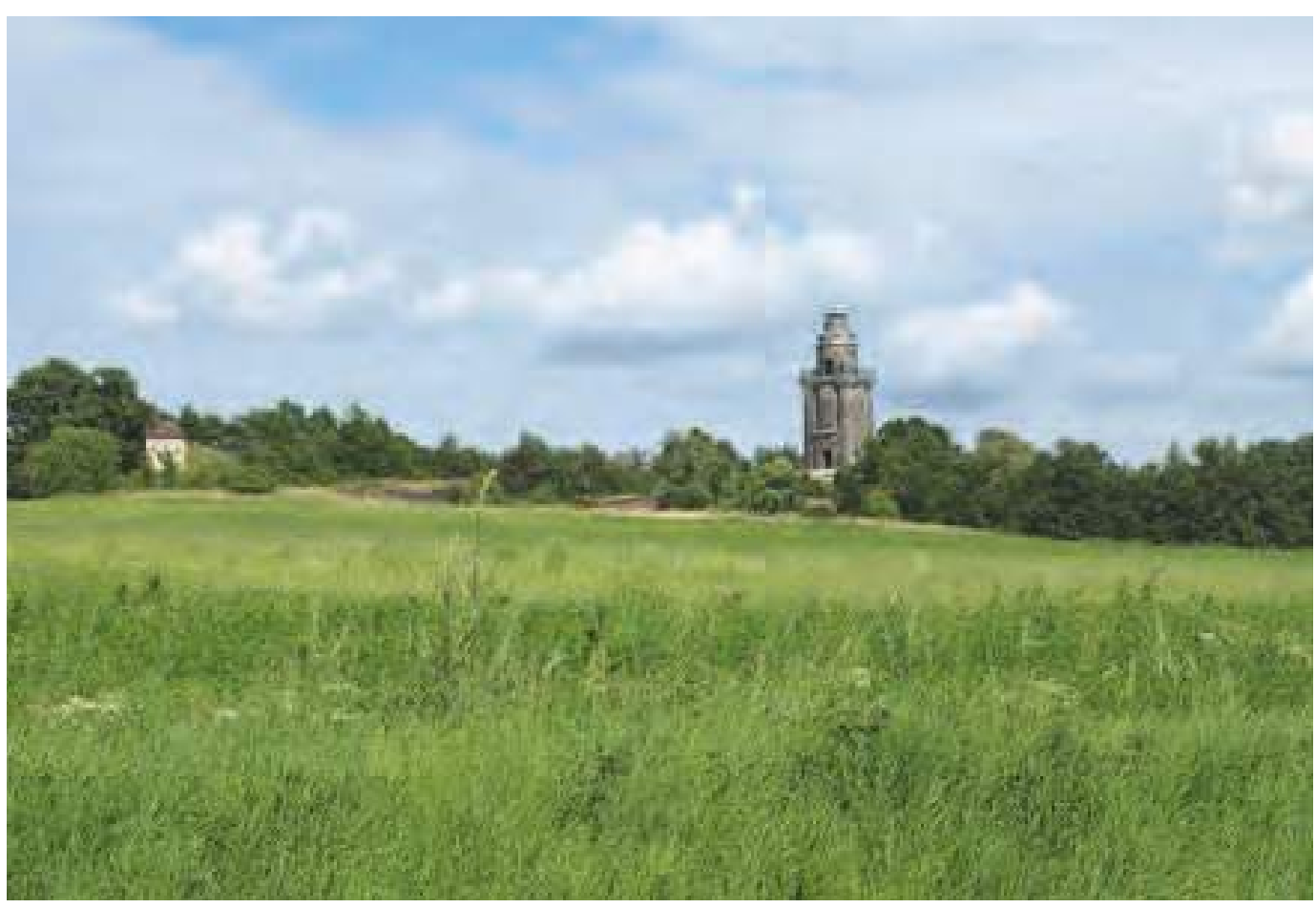


de. Für Wurzen wurde aber ein anderer Entwurf realisiert.

Die Grundsteinlegung erfolgte am 30. Juli 1908. Als Baumaterial wurde Lüptitzer Quarzporphyr und Bruchsandstein verwendet. Der Turm ist 1,50 Meter tief in den Felsen gegründet und ruht auf einer Zementbetonsohle. Er erhebt sich 18 Meter hoch auf quadratischem Grundriss mit einem pyramidenstumpfartigen Sockel, welcher über einen rampenartigen Aufstieg erreichbar ist. Der nordwestliche Eingang ist über eine Treppe begehbar. Der Bau wirkt sehr wuchtig mit kleinen Rundsäulen an den Ecken. Oberhalb des Gurtgesimses befindet sich eine Aussichtsplattform mit Geländer. Inmitten der Plattform ein kleinerer Rundbau mit Zinnenkranz und Aussichtsplattform sowie darüber erhöhte Dachabdeckung (ehemals die abschließende Feuerschale). Heute auf dem Dach eine Wetterfahne und ein Turmfalkenkasten. Ehemals über eine Holztreppe, heute Stahltreppe, erreicht man die Aussichtsgalerie. Die Gesamtkosten betrugen etwa 21.000 Mark. Am 1. April 1909 wurde der Turm feierlich eingeweiht und auch erstmals befeuert. Mehrere Tausend Besucher nahmen an der Einweihungsfeier mit Fackelzug zum Turm teil. Dann wurde er der Öffentlichkeit zugänglich gemacht.

1911 ging der Bismarckturm in das Eigentum und die Verwaltung der Stadtgemeinde Wurzen über. Am 16. April 1945 wurde der Turm beim Gefecht zwischen Amerikanern und dem Volkssturm durch mehrere Treffer stark beschädigt. Schäden an der Außenmauer und dem Turmkopf waren die Folge, ebenso wurde die Holztreppe zerstört. 1947 war die Sprengung des beschädigten Turmes geplant, die aber verhindert werden konnte. Der Turm wurde nun aus ideologischen Gründen „Wachtelbergturm“ genannt. Geplante Sanierungen fanden nicht statt, doch wurden 1954 durch erste Maßnahmen die Kriegsschäden beseitigt. Für die Öffentlichkeit blieb der Turm verschlossen. Der Rat des Kreises erwog im April 1974 die Sprengung des Turmes sowie die Verfüllung des ehemaligen Steinbruchs mit Produktionsmüll der benachbarten Wasserglasfabrik. Der Wurzener Naturschützer Klaus Zeibig setzte sich für den Turm und dessen Umgebung ein und konnte die Pläne verhindern. Von 1975 bis 1984 baute Klaus Zeibig in seiner Freizeit am Turm. 1978 fanden umfassende Sanierungsmaßnahem statt, bei der unter anderem die Feuerschale entfernt wurde. Der Turmkopf wurde umgebaut und erhielt eine neue Überdachung, wodurch die Turmhöhe auf insgesamt 19,40 Meter stieg. Die zerstörte

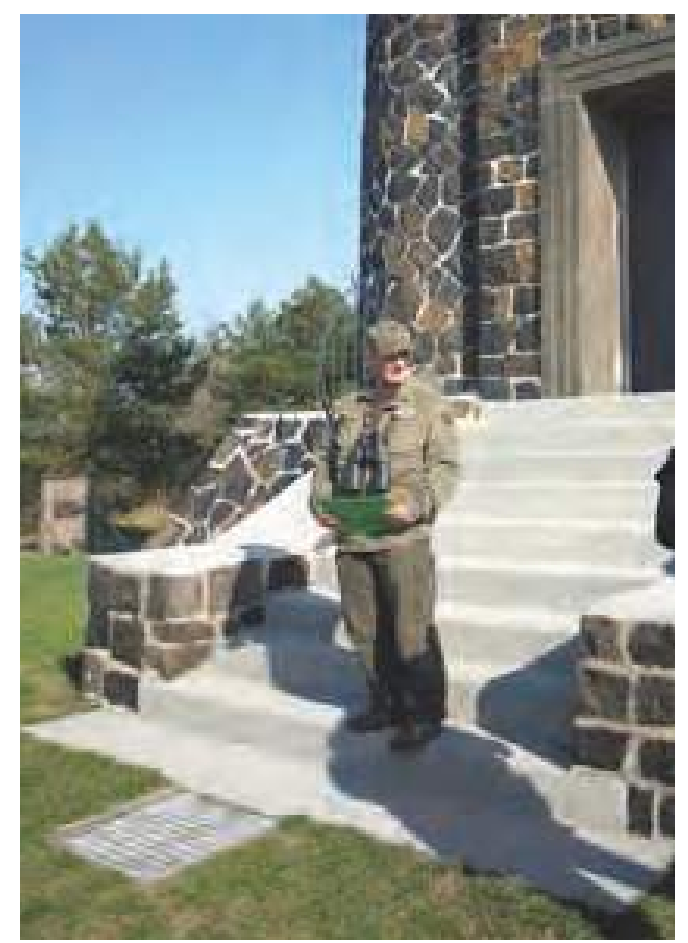

Klaus Zeibig begrüßt die Gäste am 1. April 2009 vor dem 100-jährigen Bismarckturm Foto: Wolfgang Ebert
Holztreppe wurde durch eine Stahltreppe ersetzt. 1979 wurde die heute noch existierende Wetterfahne durch einen Wurzener Kunstschmied angefertigt und auf das Dach gesetzt. Am 6. Oktober 1984 konnte der Wachtelbergturm wieder für Besucher geöffnet werden. Von da an übernahm Klaus Zeibig die regelmäßigen Turmöffnungszeiten. Weitere Sanierungen erfolgten 1992/93 und 2008. Heute befindet sich im Inneren eine Ausstellung zur Tier- und Pflanzenwelt des NSG und der Mulderegion sowie zu Baumaßnahmen am Turm. Vom Turm aus hat man bei klarer Sicht einen weiten Ausblick bis $40 \mathrm{~km}$ in die Umgebung.

Der Steinbruch unterhalb des Turmes ist, abhängig vom Niederschlag, mit Wasser gefüllt. Fehlt das Regenwasser, bleibt er trocken - wie in diesem Jahr. Dann erkennt man auf dem Grund kleine bauliche Reste. Sie stammen von der in den Jahren 1953/54 im damaligen Nationalen Aufbauwerk des Kreises Wurzen erbauten „Naturbühne am Wachtelberg“. Von den Aufbauhelfern, vorwiegend Mitgliedern der Chöre aus Wurzen und Umgebung, wurden eine Bühne und etwa eintausend im Halbkreis angeordneten Sitzplätzen geschaffen. Die Gelder kamen aus Lottomitteln und dem Staatshaushalt. An sommerlichen Abenden fanden Veranstaltungen wie Chorkonzerte, Theater-, Volkskunstund Filmvorführungen und ähnliches statt. Tausende Menschen besuchten diese Veranstaltungen. Eine große Belastung für ein Schutzgebiet! Man stellte später fest, dass die Nutzung in keinem Verhältnis zu Aufwand und Kosten stand. Ende der 1960er Jahre fand kaum noch eine 
Naturbühne am Wachtelberg im Steinbruch unterhalb des Bismarckturms, 1954 Foto: Müller (Archiv Wolfgang Ebert)

Der renaturierte Steinbruch heute Foto: Wolfgang Ebert

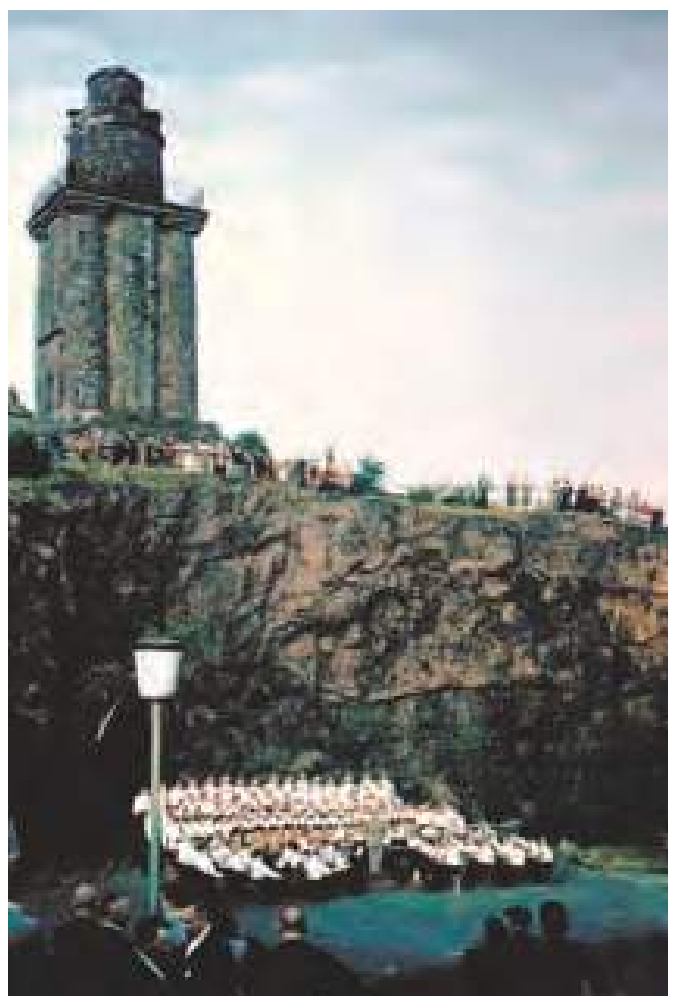

Nutzung statt und die Anlage verfiel zusehends. Neben steigendem Grundwasser waren auch Vandalismus und Diebstahl Gründe, die Anlage nach 1980 zurückzubauen und sie wieder der Natur zu überlassen.

Nach Eröffnung des Aussichtsturmes am 1. April 1909 kam zu einem starken Begängnis durch
Besucher, und somit bestand die Gefahr der Beeinträchtigung besonders der Kuhschellenbestände. Am 23. Juli 1911 erließ die Amtshauptmannschaft Grimma auf Bitten des Landesvereins Sächsischer Heimatschutz ein Betretungs- und Blumenpflückverbot für die Gipfelkuppe. 1949 und 1976 wurde der Schutzstatus als Naturdenkmal bestätigt und 1994 wurde es nochmals erweitert und als NSG festgesetzt.

Der Schutzstatus beinhaltet vor allem die Erhaltung der Trockenrasen und Felsbereiche als Lebensraum seltener Tier- und Pflanzenarten trockenwarmer Standorte. Und natürlich ist wichtigstes Anliegen die Sicherung des letzten sächsischen Vorkommens der Echten Kuhschelle (Pulsatilla vulgaris), auch Osterblume genannt. Sie gehört zu den Hahnenfußgewächsen und ist mit Anemonen nahe verwandt. Der Name geht auf die glockenähnliche Form der Blüten mancher Arten (Schelle) zurück, wobei der Tiername wohl auf das frühere Vorkommen auf beweideten Magerrasen hinweist. Es vergehen zehn bis fünfzehn Jahre, ehe die Pflanze eine Blüte zeigt. Die Kuhschelle war Blume des Jahres 1996. Ihre Pracht kann man vor allem von März bis Mai bewundern.

Das man sie bewundern kann, und das in größerer Zahl, ist nicht zuletzt das Verdienst von dem seit 1954 als Naturschützer tätigen Klaus Zeibig aus Wurzen. Seit 1965 hat er sich für die Osterblume und den Erhalt des Wachtelberges

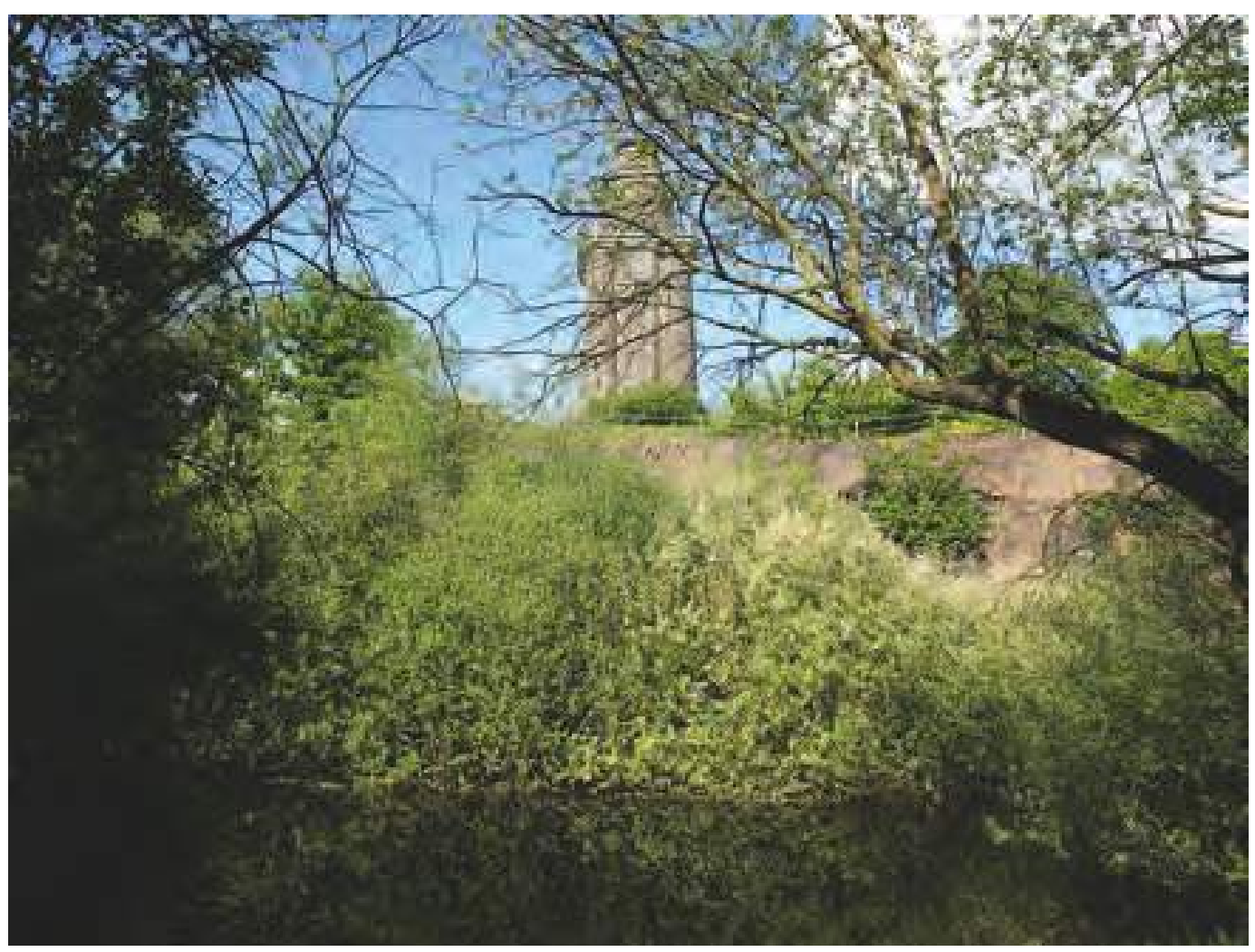




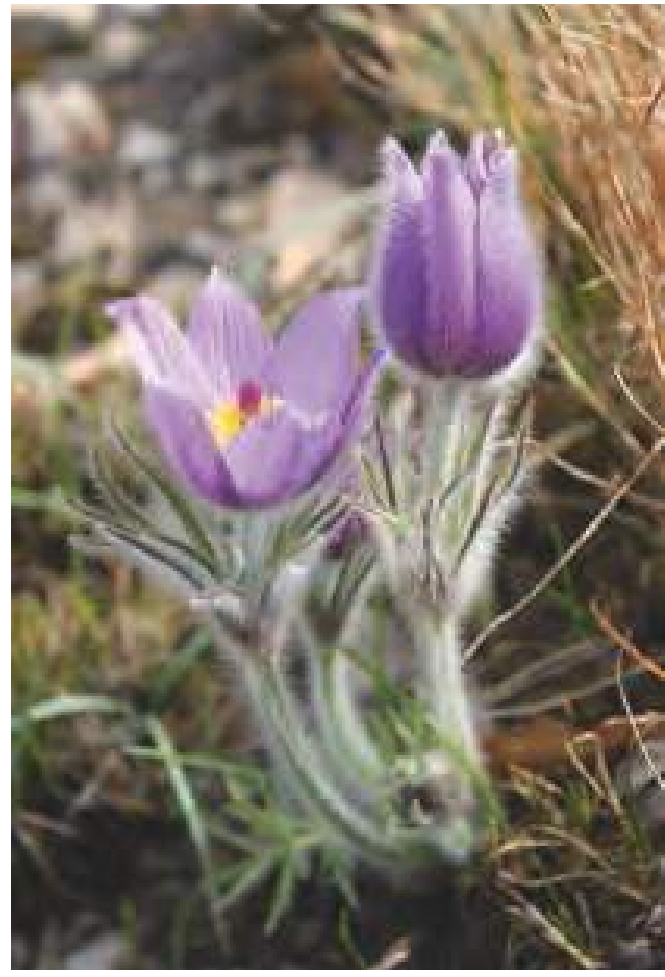

verdient gemacht. Zu Anfang der 1970er Jahre setzte er sich, gegen erhebliche politische Repressalien, erfolgreich gegen Pläne zur Sprengung des Wachtelbergturmes und die Verfüllung des Steinbruches mit Industriemüll ein. Dank seines zeitintensiven und engagierten Einsatzes konnte sich der Bestand der Echten Kuhschelle von nur 38 Pflanzen auf etwa 3.000 Exemplare in 25 Jahren erhöhen. Der Rückgang der Art wurde unter anderem durch Ausgrabungen von Kleingärtnern, Abreißen und Zertreten bei Geländespielen der Jungen Pioniere und bei Kampfgruppenübungen der DDR, Skifahren und Rodeln bei geringer Schneehöhe, Kunstdüngeranflug sowie Pflanzenschutzmittel von den umliegenden Feldern verursacht. Auch heute ist es notwendig, unbelehrbaren Zeitgenossen auf die Finger zu schauen und auf die Einhaltung des Wegegebotes zu verweisen. Als der „Herr vom Wachtelberg“ ist Klaus Zeibig weithin bekannt. Mit Führungen und Exkursionen bringt er den Besuchern das Gebiet mit seiner besonderen Flora und Fauna näher und mit regelmäßigen Pflegmaßnahmen sorgt er für den Erhalt und die Förderung verschiedener Lebensräume. Sein Engagement wurde mit dem Heimatpreis für Natur 2000 des Muldentalkreises, der „Goldenen Natur 2001“ der Deutschen Bundesstiftung Umwelt und dem Feldschlößchen-Naturschutzpreis geehrt.

Der Wachtelberg besitzt eine große Biotoptypenvielfalt auf kleinem Raum. Der Bestand der Kuhschelle hat sich stets positiv entwickelt. Im Bereich des Kuppenstandortes sind Arten zu finden, die auf der Roten Liste Sachsen vertreten sind, zum Beispiel Hainsegge, Kleiner Vogelfuß, Knolliger Hahnenfuß, KnöllchenSteinbrech, Frühlings-Spark, Strand-Grasnelke, Kartäusernelke, Gewöhnliches Bitterkraut, Hohes Fingerkraut. Auf besonnten Stellen findet man mehrere Fetthennen-Arten, Ausdauernder Knäuel, Nelken-Haferschmiele, SandVergißmeinnicht und Platthalm-Rispengras. Bei einer zunehmenden Bodentiefe folgt ein Labkraut-Straußgras-Halbtrockenrasen mit Zierlichem Schillergras, Furchen-Schafschwingel und Rispen-Flockenblume. Bei größerer Bodentiefe findet man Besenheide, Blut-Storchschnabel, verschiedene Nelkenarten und Besenginster. Zum Tal hin folgen Glatthafer-Frischwiesen. Auf der Ackerbrache im Westen kommt eine artenreiche und bunte Pflanzengesellschaft vor, während im Osten mehr Halbtrockenrasen ruderalen Charakters mit Pioniervegetation vorherrscht. Zum Mühlbach hin dominieren vor allem Röhrichte und Großseggen-Riede sowie bachbegleitend Fragmente eines Traubenkirschen-Erlen-Eschenwaldes. Außerdem dichte verbuschte Bereiche. Auf Grund dieser Naturausstattung haben sich zahlreiche Tierarten im NSG angesiedelt. Einen Steinbruch und Kleingewässer, durch Regenwasser gespeist, gibt es am Wachtelberg und das größte Standgewässer ist das ehemalige Bad "Goldenes Tälchen“ neben dem Mühlbach am Südhang des Wachtelberges.

Wie es der Name andeutet, stammt es aus den „goldenen Zeiten“, den zwanziger Jahren. Privater Unternehmergeist schuf hier durch Aufstauen des Mühlbaches 1925/26 eines der ersten, modernsten und idyllischsten Familienbäder
Echte Kuhschelle

Foto: Wikimedia, Marco Schmidt
Trockenmauern am Hang des Wachtelbergs zur Befestigung von Ackerterrassen Foto: Wolfgang Ebert

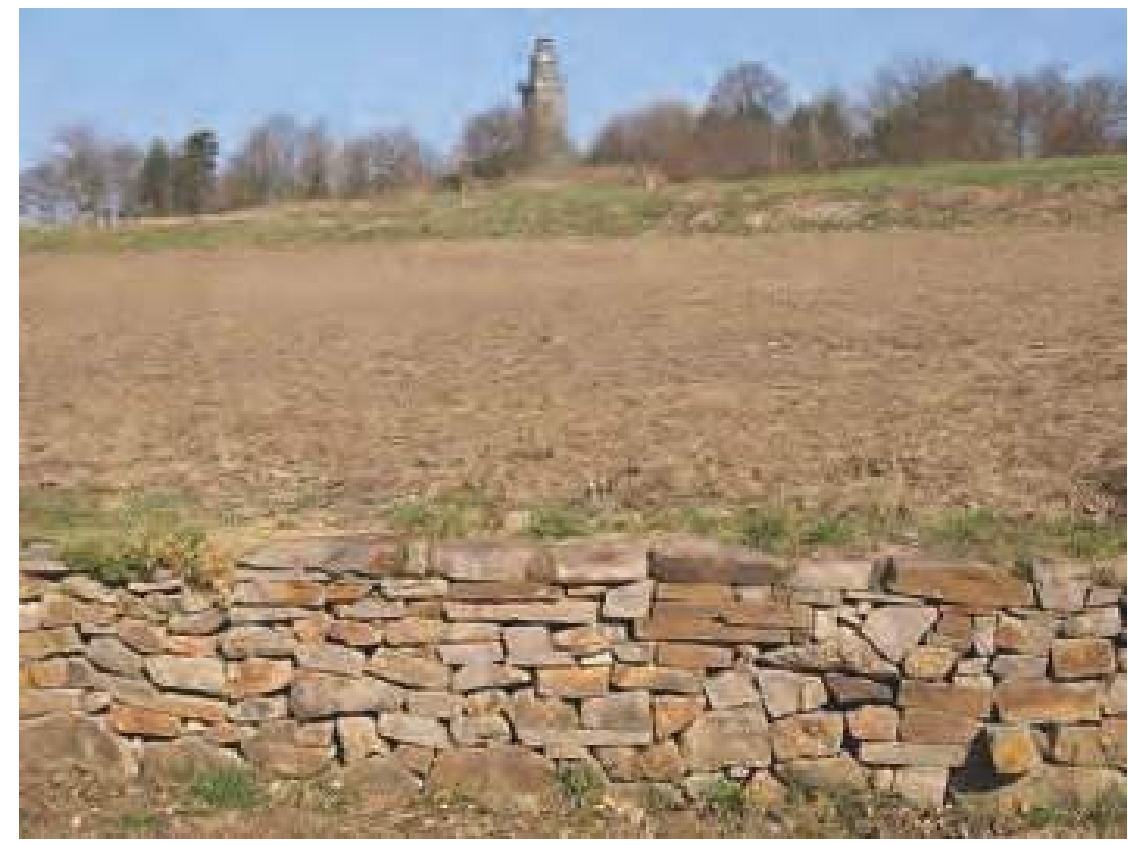


Familienbad "Goldenes Tälchen", dahinter der Wachtelberg, 1930 Foto: Archiv Wolfgang Ebert
Sachsens. Es hatte $50.000 \mathrm{~m}^{2}$ Gesamtfläche, davon $15.000 \mathrm{~m}^{2}$ Wasserfläche mit Sprungturm, Wasserrutschbahnen, Holzpodest, Schaukel mit Klettertauen und Ringen, Schwebebahn sowie große Wiesenflächen für das Sonnen- und Luftbad. Dabei auch eine Gastwirtschaft mit überdachten Kolonaden, die Unterkunft für 600 Gäste bot. Sie verfügte über eine eigene Konditorei und eine Kapelle für Nachmittags- und Abendkonzerte. Heizungsanlagen machten sogar Winteröffnungszeiten möglich, in denen man auf der Eisfläche Schlittschuh laufen konnte. Tausende kamen an den Wochenenden aus der nahen Großstadt. Auch in DDR-Zeiten wurde das Bad weiter genutzt, aber später machten sich Erhaltungsmaßnahmen notwendig. Das Bad wurde mit hohem finanziellem Aufwand von 1976 bis 1979 zu einem Naherholungszentrum der Stadt mit Sommergaststätte ausgebaut. Endgültig geschlossen wurde das Bad 1991 wegen schlechter Wasserqualität. Der Verfall beschleunigte sich durch die Nutzung als "Jugendhaus“. Die Natur hat sich inzwischen längst alles zurückgeholt und einen wertvollen Biotop geschaffen. Heute finden wir dort den Kammmolch und den Laubfrosch, außerdem auch Vorkommen von Wechselkröte und See- frosch. Ein Naturlehrpfad führt um das Gewässer. Von den Reptilien wurden im NSG Zauneidechse, Schlingnatter, Blindschleiche, Ringelnatter und Kreuzotter nachgewiesen. Am Mühlbach wurden auch schon Biber und Fischotter beobachtet. Die Insektenwelt ist reich, unter anderm wurden auf dem Wachtelberg 140 Arten Großschmetterlinge nachgewiesen, darunter Großer Schillerfalter, Großer Fuchs und Schwalbenschwanz. Von Heuschrecken wurden 17 Arten nachgewiesen, darunter die Punktierte Zartschrecke und die Blauflügelige Ödlandschrecke. Weitere Insektenvertreter sind der Laufkäfer Lebia cruxminor, Grabwespen, Wildbienenarten, Rote Waldameise und die Spinne Xerolycosa miniata (Wolfsspinnenart). Die Vogelwelt ist vor allem im Bereich des Mühlbaches vielfältig. Hier hört man Kohlund Blaumeisen, Gartenbaumläufer, Mönchsund Gartengrasmücke, Nachtigall, Zilpzalp, Rotkehlchen, Star, Pirol und andere mehr. Der Eisvogel ist ebenfalls hier heimisch. Auch Mäusebussard, Turmfalke und Rotmilan sind über den Ackerflächen um den Turm zu beobachten.

Das NSG Wachtelberg-Mühlbachtal ist eines der wertvollsten in ganz Sachsen und Teil des

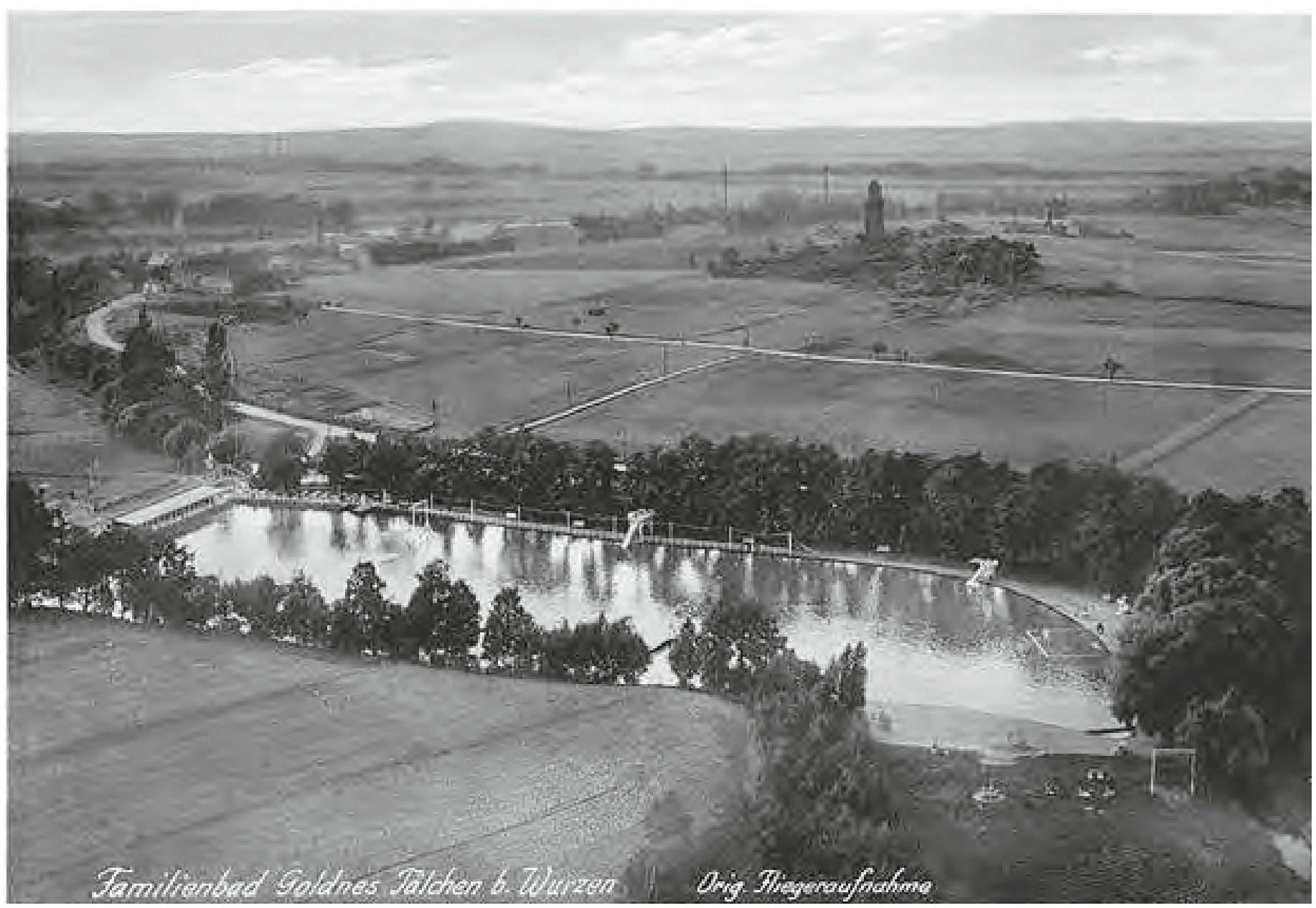



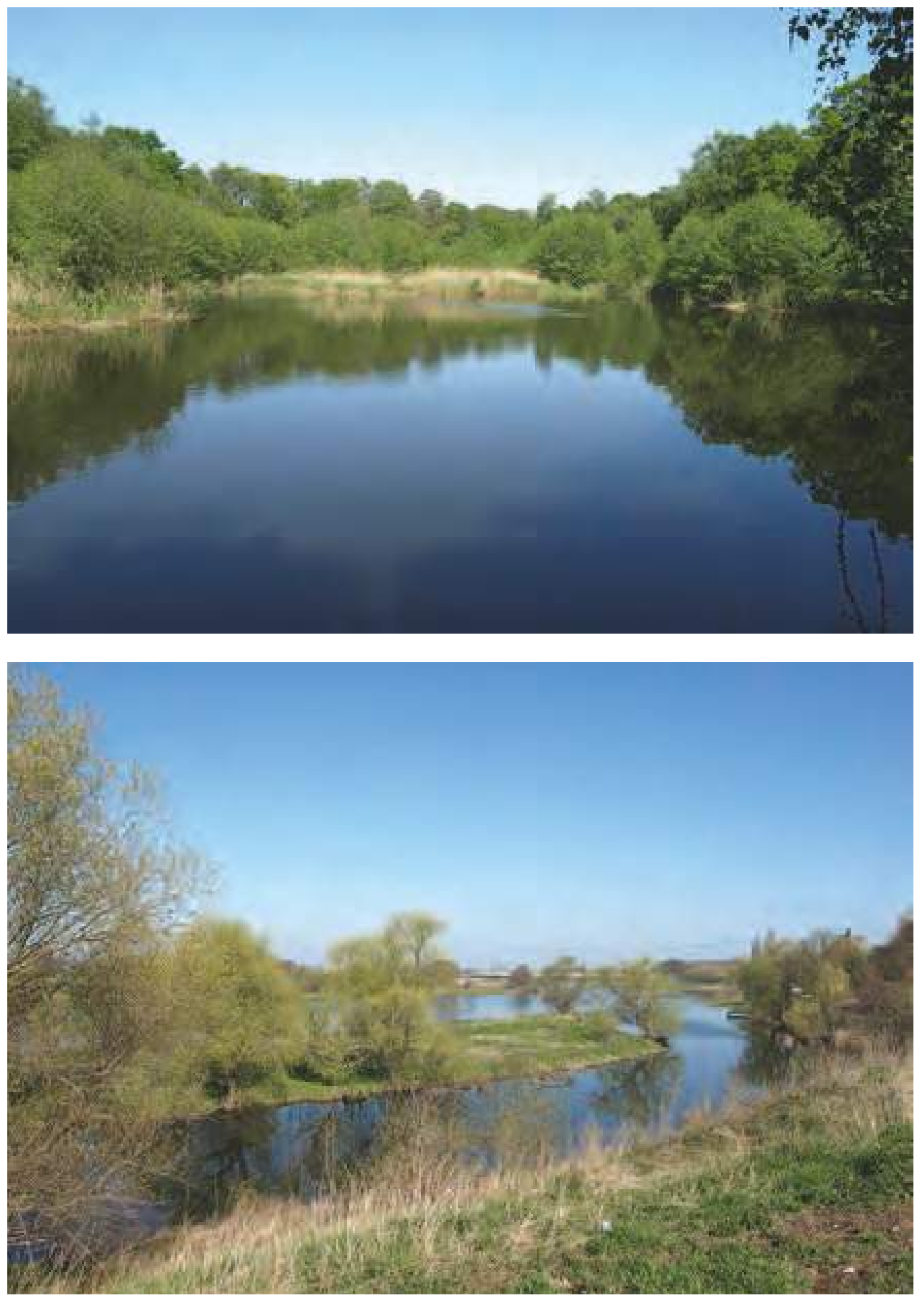

Fauna-Flora-Habitat-Gebietes „Vereinigte Mulde und Muldeauen“ des Programms „Natura 2000“ der Europäischen Union. Bis es dazu kam, war viel persönlicher Einsatz nötig, um das Schützenswerte zu erhalten. Wir alle sollten dieses Gut schützen und bewahren! Darum passen als Schlussworte auch folgende Textstellen, die ab Frühjahr 1912 durch die Amtshauptmannschaft Grimma als erklärende Verbotstafeln auf dem Wachtelberggelände angebracht worden sind: „Der Wachtelberg trägt inmitten der fruchtbaren Getreidefelder noch heute seine ursprüngliche Pflanzenwelt und zeigt uns, wie die Flora der sonnigen Hügel östlich von den Leipziger Auewäldern zusammengesetzt war, bevor der Mensch mit seinen Kulturflächen sie zerstörte. Er ist also als Naturdenkmal anzusehen, das uns wie eine wertvolle Urkunde von alten Zeiten berichtet. Dieses auch für unsere Nachkommen zu erhalten, ist unsere Pflicht."
Das ehemalige Familienbad heute. Die Natur hat sich die verwahrloste Stätte zurückgeholt. Foto: Wolfgang Ebert

Oberhalb der Wurzener

Eisenbahnbrücke mündet der Mühlbach (hier Dehnitzer Lache genannt) in die Mulde. Foto: Wolfgang Ebert

Literatur:

Wurzen und die Muldenlandschaften zwischen Dehnitz und Thallwitz. Rundblick-Information 4.

Wurzen 1978.

Arno Naumann: Die Osterblume am Wachtelberg bei Wurzen.

In: Mitteilungen des Landesvereins Sächsischer Heimatschutz 11 (1922) S. 153-158.

Joseph Richter: Führer durch Wurzen und Umgebung. Wurzen 1936

Klaus Zeibig: 70 Jahre Schutz dem Wachtelberg.

In: Der Rundblick 29 (1982),

Heft 1, S. 63-65.

Klaus Zeibig: Das Naturschutzgebiet Wachtelberg-Mühlbachtal. In:

Sächsische Heimatblätter 42 (1996), Heft 3, S. 138-140.

\section{Autor}

Jens Müller

Brandis 